\title{
A correlative cross sectional study on Manas Prakriti and psychological parameters among young females
}

\author{
Research Article
}

\author{
${\text { Divyani Soni }{ }^{*} \text {, Vandana Verma }}^{2 *}$, Shobhna Joshi ${ }^{3}$, Sangeeta Gehlot ${ }^{4}$ \\ 1. MD Scholar, 2. Assistant Professor Stage-III, 3. Professor, \\ Department of Psychology, Faculty of Social Science, \\ 4. Professor, Department of Kriya Sharir, Faculty of Ayurveda, IMS, \\ Banaras Hindu University, Varanasi, India.
}

\begin{abstract}
Background: Several scientific studies have documented the relationship among personality types, psychological traits and disorders. Manasika Prakriti, described in Ayurveda, is based on the three qualities of mind i.e Satva, Raja and Tama which determine the three Manasika Prakriti, namely, Satvika, Rajasika, and Tamasika . Among these, Satvika individuals are less prone for psychological disorders than Tamasika and Rajasika type. Few studies have been conducted on relationship of psychological parameters and Manas Prakriti, Thus it requires a large sample survey specifically in Indian population to make conclusive statements. Aim and Objective: To find an association between Manasika Prakriti and Psychological parameter i.e Stress, depression and anxiety among healthy females. Methods: A total 450 healthy females (180 Satvika, 145 Rajasika and 125 Tamasika Prakriti) were selected from different department of Banaras Hindu University, Varanasi. Assessment of Manasika Prakriti was done by using Manas Prakriti assessment inventory(MPAI) developed by Aftab A et.,al, 2018 and psychological parameters i.e stress, depression and anxiety were assessed by using Stress Scale, Beck depressions inventory II(BDI II) and Sinha's anxiety scale. Result: Significant difference was found in between Manasika Prakriti and Psychological parameters i.e. stress, depression and anxiety at the faculty level $(\mathrm{p}<0.001)$. In all the faculty Satvika Prakriti females having very low level of stress and anxiety $(63.4 \%, 34.4 \%)$ and minimal level of depression(76.6\%) while few Rajasika Prakriti females having mild level of depression(47.6\%) and severe level of anxiety(53.8\%).Tamasika Prakriti females were having higher chances of severe level of stress, depression and anxiety as compared to other Prakriti.
\end{abstract}

Key Words: Manasika Prakriti, Stress, Depression, Anxiety.

\section{Introduction}

"Ayurveda" deals through individualized approach for the maintenance of health as well as for the management of disease. The dimensions of the individualized approach are- Prakriti, Satva, Sara, Samhanan, Bala etc. Out of these dimensions, Prakriti has been most interested area for the researchers. Prakriti can be classified in to two groups i.e. Deha Prakriti and Manas Prakriti. Deha Prakriti is dependent on the permutation and relative dominance of the three Dosha Vata, Pitta and Kapha Dosha whereas Manas Prakriti by Gunas i.e. Satva, Raja and Tama. These Guna are responsible for psychological variability among individuals, which can be observed in their behavioral expressions, responses against any kind

* Corresponding Author:

Divyani Soni

PG Scholar,

Department of Kriya Sharir, Faculty of Ayurveda,

IMS, Banaras Hindu University, Varanasi, 221005,

Uttar Pradesh. India.

Email Id: divyanisoni21@gmail.com of stress and propensity towards Manasika Vikara i.e. psychological disorders.(1)

Ayurveda scholars have given due importance to social, mental and spiritual health among the several dimensions of health. As per WHO,1948 "Health is a state of complete physical, mental and social well-being and not merely the absence of disease or infirmity", Without mental health, there can be no true physical health. $(2,3)$ The previous data show that the worldwide $10 \%-20 \%$ of adolescents experience mental health disorders and $14 \%$ of the global burden of diseases is accredited to mental illness like stress, depression, anxiety, dementia, intellectual disabilities etc are growing continuously and imparting a significant effect on health.(4) Global data, 2021 estimates that Major depressive disorders are more than twice as prevalent in young women than men. The prevalence of mental illness i.e depression is higher among females (24.5\%) than males (16.3\%).(5) In an epidemiological study from India, $14.5 \%$ of adolescents were found to be suffering from anxiety disorders, majority of females (20-30\%) suffered from Major depressive disorder. $(6,7,8)$

Ayurveda has an extensive knowledge regarding the role of Satva (psyche) in health and psychotherapy. 
Today's lifestyle contributes to manifest different Manasika Vikaras i.e. Kama (desire), Krodha(anger), Lobha(greed), Moha(infatuation), Irshya(envy), Maan(pride), Mada (arrogance), Shoka(grief), Chittodvega(anxiety), Bhaya(fear), Harsh(elation) etc. Manas Prakriti determines the individual behaviour, thought process, mental activities etc. Ayurveda has described three types of Manas Prakriti i.e. Satvika, Rajasika and Tamasika, having characteristics according to their Guna. $(9,10)$ Our personality, behavior, and lifestyle all have important influences on our stress and anxiety level.(11)

A variety of personality classifications have been proposed over the past century, but in 1980 the Five-Factor Model (FFM) of personality was introduced.(12,13) These "Big Five" traits are Neuroticism, Extraversion, Conscientiousness, Agreeableness, and Openness to experience. $(14,15)$ Studies have reported that depressive disorders and anxiety are characterized by high levels of neuroticism/ negative emotionality (N/NE), and low levels of extraversion/positive emotionality(E/PE).(16,17,18,19) Many studies have found that individuals with MDD report higher levels of N/NE.(20,21,22) Hossein Ali Ghorbani Amir et.al., 2011, have reported that subjects of Type A personality have low quality of mental health in comparison to the subjects having Type B personality.(23) These data suggest the need of clinical research related to personality, stress and psychological disorders.

\section{Material and Method}

This study was conducted in the Department of Kriya Sharir, IMS , BHU during the year 2018-2019. Healthy young female students, aged 18-30 years were selected from different Faculty of Banaras Hindu University, Varanasi, after getting their written informed consent. The females suffering from chronic illness such as PCOS, hypothyroidism etc.were excluded from the study. Initially total 500 females were registered as per inclusion and exclusion criteria. 50 females were dropped from study as the complete data could not be obtained, thus analysis was done on data of 450 subjects. The participants were categorized into four groups i.e UG-Medical(BAMS, BDS and MBBS), PGMedical (Ayurvedic and Modern Medicine), UG-Social Science (home science, performing art and visual art ) and UG-Science (chemistry, mathematics, geography and zoology).

Assessment of Manasika Prakriti, stress, depression and anxiety was done by following validated and reliable scales:

1. Manas Prakriti Assessment Inventory(MPAI) (24)

2. Stress scale.(SS)(25)

3. Beck Depression Inventory II (BDI II).(26)

4. Sinha Anxiety Scale.(27)

The Analysis of data was done by using SPSS 16.0. Chi square test, One way ANOVA, Posthoc test, kruskal-Walis test, Mann-Whitney test and correlation coefficient was applied to infer the result.

\section{Observations and Results}

Among the study population, Out of 450 females, $180(40 \%)$ volunteers belonged to Satvika Prakriti while 145(32.23\%) were of Rajasika Prakriti and $125(27.77 \%)$ were of Tamasika Prakriti.

\begin{tabular}{|c|c|c|c|c|c|}
\hline Manas Prakriti $(n=450)$ & $\begin{array}{c}\text { Very low } \\
(n=210)\end{array}$ & $\begin{array}{c}\text { Low } \\
(n=141)\end{array}$ & $\begin{array}{c}\text { Moderate } \\
\quad(\mathbf{n}=\mathbf{3 9})\end{array}$ & $\begin{array}{l}\text { High } \\
(n=47)\end{array}$ & $\begin{array}{c}\text { Severe } \\
(n=13)\end{array}$ \\
\hline Satvika Prakriti $(n=180)$ & $114(63.4 \%)$ & $48(26.6 \%)$ & $16(8.9 \%)$ & $2(1.1 \%)$ & $0 *(0.0 \%)$ \\
\hline Rajasika Prakriti $(n=145)$ & $94(64.9 \%)$ & $29(20 \%)$ & $18(12.4 \%)$ & $4(2.7 \%)$ & $0 *(0.0 \%)$ \\
\hline Tamasika Prakriti $(n=125)$ & $2(1.6 \%)$ & $64(51.2 \%)$ & $5(4 \%)$ & $41(32.8 \%)$ & $13(10.4 \%)$ \\
\hline
\end{tabular}

$$
\chi 2=220.6, \quad \mathbf{p}<\mathbf{0 . 0 0 1}
$$

Very low level of stress was observed in 114(63.4\%) Satvika Prakriti individuals followed by Rajasika Prakriti 94(64.9\%) while high and severe stress level was observed in 41(32.8\%) and 13(10.4\%) Tamasika Prakriti individuals.(Table No.1)

Table No. 2 Depression level as per Manas Prakriti

\begin{tabular}{|c|c|c|c|c|}
$\begin{array}{c}\text { Manas Prakriti } \\
(\mathbf{n}=\mathbf{4 5 0})\end{array}$ & $\begin{array}{c}\text { Minimal } \\
(\mathbf{n}=\mathbf{1 9 7})\end{array}$ & $\begin{array}{c}\text { Mild } \\
(\mathbf{n = 9 8})\end{array}$ & $\begin{array}{c}\text { Moderate } \\
(\mathbf{n = 4 2})\end{array}$ & $\begin{array}{c}\text { Severe } \\
(\mathbf{n}=\mathbf{1 1 3})\end{array}$ \\
\hline Satvika Prakriti $(\mathrm{n}=180)$ & $138(76.6 \%)$ & $26(14.4 \%)$ & $8(4.3 \%)$ & $8(4.3 \%)$ \\
\hline RajasikaPrakriti $(\mathrm{n}=145)$ & $56(38.6 \%)$ & $69(47.6 \%)$ & $20(13.8 \%)$ & $0 *(0 \%)$ \\
\hline TamasikaPrakriti $(\mathrm{n}=125)$ & $3(2.4 \%)$ & $3(2.4 \%)$ & $14(11.2 \%)$ & $105(84 \%)$ \\
\hline
\end{tabular}

$\chi 2=412.4, \quad \mathbf{p}<\mathbf{0 . 0 0 1}$

The maximum females 138(76.6\%) of Satvika Prakriti have minimal level of depression while 105( 84\%) Tamasika Prakriti females were found with severe depression level. The frequency of depression level in different Manasika Prakriti was found significant $(\mathbf{p}<\mathbf{0 . 0 0 1})$ on applying chi square test.(Table No.2) 


\begin{tabular}{|c|c|c|c|c|c|}
\hline $\begin{array}{c}\text { Manas Prakriti } \\
(\mathbf{n = 4 5 0 )}\end{array}$ & $\begin{array}{c}\text { Very low } \\
(\mathbf{n = 6 3 )}\end{array}$ & $\begin{array}{c}\text { Low } \\
(\mathbf{n = 3 4 )}\end{array}$ & $\begin{array}{c}\text { Normal } \\
(\mathbf{n = 7 3 )}\end{array}$ & $\begin{array}{c}\text { High } \\
(\mathbf{n = 9 8})\end{array}$ & $\begin{array}{c}\text { Very high } \\
(\mathbf{n}=\mathbf{1 8 2})\end{array}$ \\
\hline Satvika Prakriti $(n=180)$ & $62(34.4 \%)$ & $21(11.6 \%)$ & $57(31.6 \%)$ & $25(13.8 \%)$ & $15(8.3 \%)$ \\
\hline Rajasika Prakriti $(n=145)$ & $0 *(0 \%)$ & $7(4.8 \%)$ & $12(8.2 \%)$ & $48(33.2 \%)$ & $78(53.8 \%)$ \\
\hline Tamasika Prakriti $(n=125)$ & $1(0.8 \%)$ & $6(4.8 \%)$ & $4(3.2 \%)$ & $25(20 \%)$ & $89(71.2 \%)$ \\
\hline & & $\chi 2=23.69, \quad \mathbf{P}<\mathbf{0 . 0 0 1}$ & &
\end{tabular}

62(34.4\%) females of Satvika Prakriti were found with very low level of anxiety, followed by 73(53.8\%) of Rajasika Prakriti and 89(71.2\%) of Tamasika Prakriti females were in severe depression.(Table No.3)

Table No. 4 Correlation analysis of Stress, Depression and Anxiety as per Manas Prakriti

\begin{tabular}{|c|c|c|c|c|}
\hline Manas Prakriti $(n=450)$ & & Stress & Depression & Anxiety \\
\hline \multirow{3}{*}{ Satvika Prakriti $(n=180)$} & Stress & 1 & $\mathrm{r}=0.309^{* *}, \mathrm{p}<0.001$ & $\mathrm{r}=0.544^{* *}, \mathrm{p}<0.001$ \\
\hline & Depression & $\mathrm{r}=0.309^{* *}, \quad \mathrm{p}<0.001$ & 1 & $\mathrm{r}=0.396^{* *}, \mathrm{p}<0.001$ \\
\hline & Anxiety & $\mathrm{r}=0.544 * *, \quad \mathbf{p}<\mathbf{0 . 0 0 1}$ & $\mathrm{r}=0.396^{* *}, \mathrm{p}<0.001$ & 1 \\
\hline \multirow{3}{*}{ Rajasika Prakriti (n=145) } & Stress & 1 & $\mathrm{r}=0.17^{*}, \mathrm{p}=0.041$ & $\mathrm{r}=0.316^{* *}, \mathrm{p}<0.001$ \\
\hline & Depression & $\mathrm{r}=0.17^{*}, \mathrm{p}=0.041$ & 1 & $\mathrm{r}=0.142, \mathrm{p}=0.09$ \\
\hline & Anxiety & $\mathrm{r}=-0.316^{* *}, \mathrm{p}<0.001$ & $\mathrm{r}=0.142, \mathrm{p}=0.09$ & 1 \\
\hline \multirow{3}{*}{ Tamasika Prakriti $(n=125)$} & Stress & 1 & $\mathrm{r}=0.008, \mathrm{p}=0.934$ & $\mathrm{r}=0.112, \mathrm{p}=0.213$ \\
\hline & Depression & $\mathrm{r}=0.008, \quad \mathrm{p}=0.934$ & 1 & $\mathrm{r}=0.276^{* *}, \mathrm{p}=0.002$ \\
\hline & Anxiety & $\mathrm{r}=0.112, \mathrm{p}=0.213$ & $\mathrm{r}=0.276^{* *}, \mathrm{p}=0.002$ & 1 \\
\hline
\end{tabular}

The interrelationship among the three parameters i.e stress, depression and anxiety with Satvika, Rajasika and Tamasika Prakriti ranging from -0.316 to 0.544. In Satvika Prakriti females stress was significantly positively correlated with depression $\left(\mathrm{r}=0.309^{* *}, \mathbf{p}<\mathbf{0 . 0 0 1}\right)$, anxiety $\left(\mathrm{r}=0.544^{* *}, \mathbf{p}<\mathbf{0 . 0 0 1}\right)$ although depression was also significantly positively correlated with anxiety $\left(\mathrm{r}=0.396^{* *}, \mathbf{p}<\mathbf{0 . 0 0 1}\right)$. In Rajasika Prakriti females stress is significantly positively correlated with depression $\left(\mathrm{r}=0.17^{*}, \mathbf{p}=\mathbf{0 . 0 4 1}\right)$ and stress is significantly negatively correlated with anxiety $\left(\mathrm{r}=-0.316^{* *}, \mathbf{p}<\mathbf{0 . 0 0 1}\right)$ although depression is positively correlated with anxiety $(\mathrm{r}=0.142, \mathrm{p}=0.09)$. In Tamasika Prakriti volunteers depression is significantly positively correlated with anxiety $\left(\mathrm{r}=0.276^{* *}, \mathbf{p}=\mathbf{0 . 0 0 2}\right)$. (Table No.4)

Table No. 5 Stress, depression and anxiety score in young females as per Manas Prakriti

\begin{tabular}{|c|c|c|c|}
\hline Manasika Prakriti $(n=450)$ & Stress Score & Depression score & Anxiety Score \\
\hline \multicolumn{4}{|c|}{ Mean \pm S.D } \\
\hline Satvika Prakriti $(n=180)$ & $25.86 \pm 9.99$ & $10.19 \pm 8.48$ & $28.06 \pm 13.88$ \\
\hline Rajasika Prakriti (n=145) & $26.81 \pm 10.62$ & $14.78 \pm 4.90$ & $49.41 \pm 12.96$ \\
\hline Tamasika Prakriti $(n=125)$ & $42.85 \pm 11.41$ & $41.51 \pm 11.73$ & $53.99 \pm 13.57$ \\
\hline
\end{tabular}

Test Applied

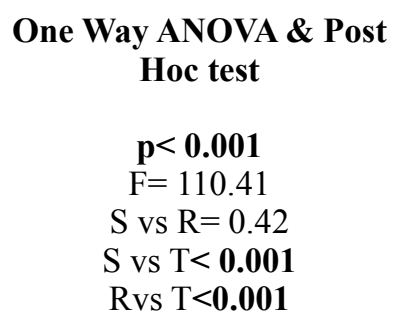

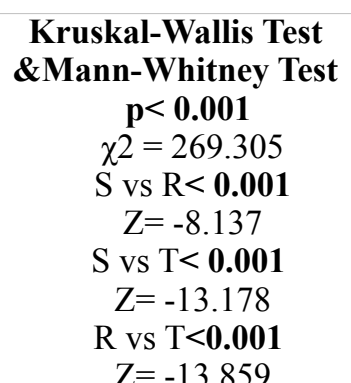
One Way ANOVA \& Post
Hoc test
$\mathbf{p}<\mathbf{0 . 0 0 1}$
$\mathrm{F}=166.87$

$\mathrm{S}$ vs $\mathrm{R}<\mathbf{0 . 0 0 1}$

$\mathrm{S}$ vs $\mathrm{T}<\mathbf{0 . 0 0 1}$

$\mathrm{R}$ vs $\mathrm{T}<\mathbf{0 . 0 0 1}$

The Mean \pm S.D score of stress, depression and anxiety in Tamasika Prakriti individuals were $42.85 \pm 11.41$, $41.51 \pm 11.73,53.99 \pm 13.57$ respectively. On applying One way ANOVA test in the stress and anxiety score was found significant $(\mathbf{p}<\mathbf{0 . 0 0 1})$ as per Manasika Prakriti. On applying Kruskal-Wallis test on depression score as per Manasika Prakriti, it was found significant $(\mathrm{p}<0.001)$.(Table No.5). 
Divyani Soni et.al., A correlative cross sectional study on Manas Prakriti and psychological parameters among young females

Table No. 6 Manas Prakriti and stress level as per Faculty

\begin{tabular}{|c|c|c|c|c|}
\hline & $\begin{array}{l}\text { Stress } \\
\text { Level }\end{array}$ & Satvika Prakriti & Rajasika Prakriti & Tamasika Prakriti \\
\hline \multirow{5}{*}{$\begin{array}{c}\text { UG (Medical) } \\
\text { Satvika=44 } \\
\text { Rajasika }=21 \\
\text { Tamasika }=15 \\
\text { Total }=80\end{array}$} & Very low $(n=36)$ & $30(83.4 \%)$ & $5(13.8 \%)$ & $1(2.8 \%)$ \\
\hline & Low $(n=26)$ & $11(42.3 \%)$ & $10(38.5 \%)$ & $5(19.2 \%)$ \\
\hline & Moderate $(n=8)$ & $3(37.5 \%)$ & $5(62.5 \%)$ & $0 *(0.0 \%)$ \\
\hline & High $(n=7)$ & $0 *(0.0 \%)$ & $1(14.3 \%)$ & $6(85.7 \%)$ \\
\hline & Severe $(n=3)$ & $0 *(0.0 \%)$ & $0 *(0.0 \%)$ & $3(100 \%)$ \\
\hline \multicolumn{5}{|c|}{$\chi 2=54.413, \mathbf{p}<\mathbf{0 . 0 0 1}$} \\
\hline \multirow{5}{*}{$\begin{array}{c}\text { PG (Medical) } \\
\text { Satvika }=66 \\
\text { Rajasika= } 35 \\
\text { Tamasika }=24 \\
\text { Total }=125\end{array}$} & Very low $(n=57)$ & $48(84.2 \%)$ & $8(14 \%)$ & $1(1.8 \%)$ \\
\hline & Low $(n=33)$ & $13(39.4 \%)$ & $14(42.4 \%)$ & $6(18.2 \%)$ \\
\hline & Moderate $(n=18)$ & $5(27.8 \%)$ & $11(61.1 \%)$ & $2(11.1 \%)$ \\
\hline & High $(n=11)$ & $0 *(0 \%)$ & $2(18.2 \%)$ & $9(81.8 \%)$ \\
\hline & Severe $(n=6)$ & $0 *(0 \%)$ & $0 *(0 \%)$ & $6(100 \%)$ \\
\hline \multicolumn{5}{|c|}{$\chi 2=90.946, \quad \mathbf{p}<\mathbf{0 . 0 0 1}$} \\
\hline \multirow{5}{*}{$\begin{array}{c}\text { UG (Social Science) } \\
\text { Satvika=48 } \\
\text { Rajasika= } 52 \\
\text { Tamasika }=48 \\
\text { Total }=148\end{array}$} & Very low $(n=73)$ & $27(37 \%)$ & $46(63 \%)$ & $0 *(0 \%)$ \\
\hline & $\operatorname{Low}(n=52)$ & $13(25 \%)$ & $4(7.7 \%)$ & $35(67.3 \%)$ \\
\hline & Moderate $(n=9)$ & $7(77.8 \%)$ & $1(11.1 \%)$ & $1(11.1 \%)$ \\
\hline & High $(n=12)$ & $1(8.3 \%)$ & $1(8.3 \%)$ & $10(83.4 \%)$ \\
\hline & Severe $(n=2)$ & $0 *(0 \%)$ & $0 *(0 \%)$ & $2(100 \%)$ \\
\hline \multicolumn{5}{|c|}{$\chi 2=98.624, \mathbf{p}<\mathbf{0 . 0 0 1}$} \\
\hline \multirow{5}{*}{$\begin{array}{c}\text { UG (Science) } \\
\text { Satvika }=\mathbf{2 2} \\
\text { Rajasika }=\mathbf{3 7} \\
\text { Tamasika }=\mathbf{3 8} \\
\text { Total }=\mathbf{9 7}\end{array}$} & Very low $(n=44)$ & $9(20.5 \%)$ & $35(79.5 \%)$ & $0 *(0 \%)$ \\
\hline & $\operatorname{Low}(n=30)$ & $11(36.7 \%)$ & $1(3.3 \%)$ & $18(60 \%)$ \\
\hline & Moderate $(n=4)$ & $1(25 \%)$ & $1(25 \%)$ & $2(50 \%)$ \\
\hline & High $(n=17)$ & $1(5.9 \%)$ & $0 *(0 \%)$ & $16(94.1 \%)$ \\
\hline & Severe $(n=2)$ & $0 *(0 \%)$ & $0 *(0 \%)$ & $2(100 \%)$ \\
\hline & & $\chi 2=77.659$ & & \\
\hline
\end{tabular}

In the UG-Medical, 30(83.4\%) subjects of Satvika Prakriti felt very low level of stress,while Tamasika Prakriti subjects having high level of stress. Among PG-Medical females, 14 (42.4\%) of the Rajasika Prakriti subjects were found with low level of stress and 9(81.8\%) Tamasika Prakriti individuals felt high stress. Among UGSocial Science, Tamasika Prakriti females were having low level of stress. In UG-Science, 35(79.5\%) Rajasika Prakriti females constitute very low level of stress followed by 16(94.1\%) Tamasika Prakriti individuals with high level of stress.(Table No.6)

Table No. 7 Manas Prakriti and depression level as per Faculty

\begin{tabular}{|c|c|c|c|c|}
\hline & Depression Level & Satvika Prakriti & Rajasika Prakriti & Tamasika Prakriti \\
\hline \multirow{4}{*}{$\begin{array}{c}\text { UG (Medical) } \\
\text { Satvika }=44 \\
\text { Rajasika }=21 \\
\text { Tamasika }=15 \\
\text { Total }=80\end{array}$} & Minimal ( $n=46)$ & $36(78.3 \%)$ & $9(19.6 \%)$ & $1(2.1 \%)$ \\
\hline & Mild (n= 11) & $4(36.4 \%)$ & $7(63.6 \%)$ & $0 *(0 \%)$ \\
\hline & Moderate $(n=11)$ & $3(27.3 \%)$ & $5(45.5 \%)$ & $3(27.2 \%)$ \\
\hline & Severe $(n=12)$ & $1(8.3 \%)$ & $0 *(0 \%)$ & $11(91.7 \%)$ \\
\hline \multicolumn{5}{|c|}{$\chi 2=66.102, \quad \mathbf{p}<\mathbf{0 . 0 0 1}$} \\
\hline \multirow{4}{*}{$\begin{array}{c}\text { PG (Medical) } \\
\text { Satvika }=66 \\
\text { Rajasika }=35 \\
\text { Tamasika }=24 \\
\text { Total }=125\end{array}$} & Minimal $(n=69)$ & $54(78.3 \%)$ & $14(20.3 \%)$ & $1(1.4 \%)$ \\
\hline & Mild $(n=25)$ & $9(36 \%)$ & $14(56 \%)$ & $2(8 \%)$ \\
\hline & Moderate (n=18) & $2(11.1 \%)$ & $7(38.9 \%)$ & $9(50 \%)$ \\
\hline & Severe $(n=13)$ & $1(7.7 \%)$ & $0 *(0 \%)$ & $12(92.3 \%)$ \\
\hline \multicolumn{5}{|c|}{$\chi 2=91.648, \quad \mathbf{p}<\mathbf{0 . 0 0 1}$} \\
\hline \multirow{4}{*}{$\begin{array}{c}\text { UG (Social Science) } \\
\text { Satvika }=\mathbf{4 8} \\
\text { Rajasika }=52 \\
\text { Tamasika }=48 \\
\text { Total }=\mathbf{1 4 8}\end{array}$} & Minimal $(n=48)$ & $29(60.4 \%)$ & $18(37.5 \%)$ & $1(2.1 \%)$ \\
\hline & Mild $(n=41)$ & $11(26.8 \%)$ & $30(73.2 \%)$ & $0 *(0 \%)$ \\
\hline & Moderate $(n=8)$ & $3(37.5 \%)$ & $4(50 \%)$ & $1(12.5 \%)$ \\
\hline & Severe $(n=51)$ & $5(9.8 \%)$ & $0 *(0 \%)$ & $46(90.2 \%)$ \\
\hline \multicolumn{5}{|c|}{$\chi^{2}=1.359, \quad \mathbf{p}<\mathbf{0 . 0 0 1}$} \\
\hline \multirow{4}{*}{$\begin{array}{c}\text { UG (Science) } \\
\text { Satvika }=\mathbf{2 2} \\
\text { Rajasika }=\mathbf{3 7} \\
\text { Tamasika }=\mathbf{3 8} \\
\text { Total }=\mathbf{9 7}\end{array}$} & Minimal $(n=34)$ & $19(55.9 \%)$ & $15(44.1 \%)$ & $0 *(0 \%)$ \\
\hline & Mild $(n=21)$ & $2(9.5 \%)$ & $18(85.7 \%)$ & $1(4.8 \%)$ \\
\hline & Moderate $(n=5)$ & $0^{*}(0 \%)$ & $\begin{array}{c}4 \\
(80 \%)\end{array}$ & $1(20 \%)$ \\
\hline & Severe $(n=37)$ & $1(2.7 \%)$ & $0^{*}(0 \%)$ & $36(97.3 \%)$ \\
\hline & & $=1.07, \quad \mathbf{p}<\mathbf{0 . 0}$ & & \\
\hline
\end{tabular}

In the UG- Medical, 36 (78.3\%) of the Satvika Prakriti females felt minimal depression and 11(91.7\%) of the Tamasika Prakriti subjects were having severe depression. Among PG-Medical, minimal depression was found in 
54(78.3\%) Satvika Prakriti females and severe depression level was maximum in 12(92.3\%) Tamasika Prakriti individuals. In UG-Social Science, mild depression was reported in 30(73.2\%) Rajasika Prakriti subjects. In UGScience, 36(97.3\%) Tamasika Prakriti females felt severe depression.(Table No.7)

Table No.8 Manas Prakriti and anxiety level as per Faculty

\begin{tabular}{|c|c|c|c|c|}
\hline & $\begin{array}{c}\text { Anxiety } \\
\text { Level }\end{array}$ & $\begin{array}{l}\text { Satvika } \\
\text { Prakriti } \\
(\mathrm{n}=180)\end{array}$ & $\begin{array}{c}\text { Rajasika } \\
\text { Prakriti } \\
(\mathrm{n}=145)\end{array}$ & $\begin{array}{c}\text { Tamasika } \\
\text { Prakriti } \\
(\mathrm{n}=125)\end{array}$ \\
\hline \multirow{5}{*}{$\begin{array}{c}\text { UG (Medical) } \\
\text { Satvika }=44 \\
\text { Rajasika }=\mathbf{2 1} \\
\text { Tamasika }=15 \\
\text { Total }=\mathbf{8 0}\end{array}$} & Very low $(n=22)$ & $21(95.5 \%)$ & $0 *(0 \%)$ & $1(4.5 \%)$ \\
\hline & Low $(n=7)$ & $2(28.6 \%)$ & $4(57.1 \%)$ & $1(14.3 \%)$ \\
\hline & Normal $(n=17)$ & $10(58.8 \%)$ & $6(35.3 \%)$ & $1(5.9 \%)$ \\
\hline & High $(n=20)$ & $7(35 \%)$ & $7(35 \%)$ & $6(30 \%)$ \\
\hline & Very high $(n=14)$ & $4(28.6 \%)$ & $4(28.6 \%)$ & $6(42.9 \%)$ \\
\hline \multicolumn{5}{|c|}{$\chi 2=29.807, \mathbf{p}<\mathbf{0 . 0 0 1}$} \\
\hline \multirow{5}{*}{$\begin{array}{c}\text { PG (Medical) } \\
\text { Satvika=66 } \\
\text { Rajasika }=35 \\
\text { Tamasika }=24 \\
\text { Total }=125\end{array}$} & Very low $(n=26)$ & $26(100 \%)$ & $0 *(0 \%)$ & $0 *(0 \%)$ \\
\hline & Low $(n=17)$ & $11(64.8 \%)$ & $3(17.6 \%)$ & $3(17.6 \%)$ \\
\hline & Normal $(n=26)$ & $18(69.3 \%)$ & $5(19.2 \%)$ & $3(11.5 \%)$ \\
\hline & High $(n=30)$ & $8(26.7 \%)$ & $19(63.3 \%)$ & $3(10 \%)$ \\
\hline & Very high $(n=26)$ & $3(11.5 \%)$ & $8(30.8 \%)$ & $15(57.7 \%)$ \\
\hline \multicolumn{5}{|c|}{$\chi 2=74.307, \mathbf{p}<\mathbf{0 . 0 0 1}$} \\
\hline \multirow{5}{*}{$\begin{array}{c}\text { UG (Social } \\
\text { Science) } \\
\text { Satvika=48 } \\
\text { Rajasika }=52 \\
\text { Tamasika }=48 \\
\text { Total=148 }\end{array}$} & Very low $(n=10)$ & $10(100 \%)$ & $0 *(0 \%)$ & $0 *(0 \%)$ \\
\hline & Low $(n=4)$ & $3(75 \%)$ & $0 *(0 \%)$ & $1(25 \%)$ \\
\hline & Normal $(n=22)$ & $21(95.5 \%)$ & $1(4.5 \%)$ & $0 *(0 \%)$ \\
\hline & High $(n=27)$ & $8(29.6 \%)$ & $11(40.8 \%)$ & $8(29.6 \%)$ \\
\hline & $\operatorname{Very} \operatorname{high}(n=85)$ & $6(7.0 \%)$ & $40(47.1 \%)$ & $39(45.9 \%)$ \\
\hline \multicolumn{5}{|c|}{$\chi 2=89.904, \quad \mathbf{p}<\mathbf{0 . 0 0 1}$} \\
\hline \multirow{5}{*}{$\begin{array}{c}\text { UG (Science) } \\
\text { Satvika=22 } \\
\text { Rajasika }=\mathbf{3 7} \\
\text { Tamasika }=\mathbf{3 8} \\
\text { Total }=\mathbf{9 7}\end{array}$} & Very low $(n=5)$ & $5(100 \%)$ & $0 *(0 \%)$ & $0 *(0 \%)$ \\
\hline & $\operatorname{Low}(n=6)$ & $5(83.3 \%)$ & $0 *(0 \%)$ & $1(n=16.7 \%)$ \\
\hline & Normal $(n=8)$ & $8(100 \%)$ & $0 *(0 \%)$ & $0 *(0 \%)$ \\
\hline & High $(n=21)$ & $2(9.5 \%)$ & $11(53.4 \%)$ & $8(38.1 \%)$ \\
\hline & Very high $(n=57)$ & $2(3.5 \%)$ & $26(45.6 \%)$ & $29(50.9 \%)$ \\
\hline
\end{tabular}

Among UG-Medical, 6(42.8\%) of Tamasika Prakriti individuals were having very high level of anxiety. In PG-Medical, 15(57.7\%) subjects of Tamasika Prakriti constitute high level of anxiety. In UG-Social Science, 40(47.1\%) Rajasika Prakriti and 39(45.9\%) Tamasika Prakriti females were having high level of anxiety. In UG-Science, $29(50.9 \%)$ of the Tamasika Prakriti individuals were having high level of anxiety. (Table No.8).

\section{Discussion}

The present research work is an observational cross sectional survey study, aimed to explore the relationship between Manas Prakriti and certain psychological traits. Several studies had shown that females had higher incidence of stress, depression and anxiety. $(28,29)$ In this study only female volunteers were selected because females are more prone to psychological illness, however less data and studies are found in females especially in India, even till date the psychological health of females is not taken into much consideration. In this study the collected data shows that the level of stress, depression and anxiety were found under severe category in some females. This was one time data collection so it may be temporary due to some circumstances but all the females who have severe level of stress, depression and anxiety were suggested to concern psychology department for counseling and further screening.
The findings showed that out of 180 Satvika Prakriti volunteers, $63.4 \%$ predominantly correspond to very low level of stress similarly $76.6 \%$ females having minimal level of depression and $34.4 \%$ females show very low level of anxiety. This seems to be justified because Satvika Prakriti individuals having the dominance of Satva Guna and having characteristics like Jitatmaanam(controlling over senses), Kaamkrodhalobha manmohairshyarahita (Devoid of attachment, anger, greed, conceit, confusion and envy), Gyansampanna(full of knowledge).(30) Out of total 145 females of the Rajasika Prakriti, 64.9\% having very low stress level similarly $38.6 \%$ females having minimal depression and $47.6 \%$ felt mild depression but $33.2 \%$ female having high level of anxiety and $53.8 \%$ felt severe anxiety. It is empirically significant and found textually that Rajasika Prakriti individuals endorsed the attributes of being passionate, desirous, performed actions without doing analysis. Out of 125 Tamasika Prakriti females, $32.8 \%$ and $10.4 \%$ having, high and severe level of stress respectively similarly $84 \%$ females having severe level of depression and $71.2 \%$ females also show the severe level of anxiety. Tamasika Prakriti individuals were more prone to high and severe stress, depression and anxiety due to the dominance of Tama Guna and the traits like Vishaditwam(Unhappiness), Nastikya(atheim), Adharmasheela (Iniquitous).(31)

In this study only Ekala Prakriti individuals were included and the significant $(\mathrm{p}<0.001)$ association 
between the Manasika Prakriti with stress, depression and anxiety was found. The Tamasika Prakriti were more prone to stress, depression and anxiety followed by Rajasika and Satvika females. A study conducted by Sharma R, 2018, considering both sex reported that Rajas Pradhan Prakriti individuals are more prone for stress.(32) A study by Das K, 2011, showed a different result that Raja Tamasika Prakriti subjects were having very severe depression. The difference in result may be due to Ekala and Dwandaja Manas Prakriti categorization and application of different assessment tool.(33)

The findings of the study show that association of the Manasika Prakriti with stress, depression and anxiety score at faculty level was found significant. Similar previous study reported that anxiety, depression and stressful life events among medical students were found to be significant.(34) The present study confirms the findings of earlier researchers, who have reported similar results.(35) In the UG-Social Science (63\%) and Science faculty $(79.3 \%)$, the females belonging to Rajasika Prakriti felt very low level of stress, however high level of stress was reported in $94.1 \%$ of Tamasika Prakriti females. Similar study has been reported that the students of Art Faculty were having higher stress. (36) A previous study reported that medical students were more prone to depression especially females.(37) The findings of the present study on depression level in different faculty female students are concurrent with the previous studies. $(38,39)$ Although in these studies subjects of both sex were included.

\section{Conclusion}

The study empirically validated the incidence of Satvika, Rajasika and Tamasika Prakriti females. This study shows significant relationship between the Manasika Prakriti and Psychological parameter i.e. stress, depression and anxiety as per faculty level in which Satvika Prakriti females were having very low level of stress, anxiety and depression while few Rajasika Prakriti females were having mild level of depression and severe level of anxiety. The Tamasika Prakriti females were having more chances of high and severe level of stress, depression and anxiety.

\section{Source of funding- Nil.}

Conflict of Interest- None declared.

Ethical Clearance- IMS, BHU (vide letter No. Dean/ 2019/EC/1306).

Acknowledgement- I would like to extend my sincere thanks to the participants of this study and the Department of Kriya Sharir, Psychology of BHU, Varanasi.

\section{Reference}

1. Sharma RK, \& Dash B. Charaka Samhita English translation. Reprint edition.Varanasi; Chowkhambha Sanskrit Series office;2018: ISBN: 978-81-7080-012-9
2. World health Organization.1948: http:// www.publichealth.com.ng.

3. Kolappa K, Henderson DC, Kishore SP, No physical health without mental health: lessons unlearned?, Bulletin of the World Health Organization, 2013; 91(1):3-3A. https://doi.org/ 10.2471/BLT.12.115063

4. Srivastava K, Medical AF, Concept of personality: Indian perspective. Industrial Psychiatry Journal, $2019 ; 21(2): 89-93$. https://doi.org/ 10.4103/0972-6748.119586

5. Whiteford HA, Degenhardt L, Rehm J, et al, Global burden of disease attributable to mental and substance use disorders, findings from the Global Burden of Disease Study, 2010. Lancet (London, England), 2013;382(9904):1575-1586. https:// doi.org/10.1016/S0140-6736(13)61611-6

6. Nair MK, Russell PS, Mammen P, et al, The epidemiology of Anxiety Disorders among adolescents in a rural community population in India, Indian journal of pediatrics, 2013;80(2):144 148. https://doi.org/10.1007/s12098-013-1097-5

7. Albert PR, Why is depression more prevalent in women?, Journal of psychiatry \& neuroscience, 2015;40(4):219-221. https://doi.org/10.1503/ jpn. 150205

8. Hendrick V, Altshule L, Cohen L, et al, Evaluation of mental health and depression during pregnancy: position paper, Psychopharmacology bulletin, 1998; 34(3): 297-299.

9. Sharma RK, \& Dash B. Charaka Samhita English translation. Reprint edition.Varanasi; Chowkhambha Sanskrit Series office;2018: ISBN: 978-81-7080-012-9

10. Murthy KR. Ashtanga Sangraha English translation.6th edition.Varanasi ; Chowkhambha Orientalia; 2017.

11. Rastogi MR, Triguna and Psychological Wellbeing, Indian Journal of Community Psychology, 2004; 1:115-124

12. Digman JM, The Developing Structure of Temperament and Personality from Infancy to Adulthood. Hillsdale, NJ: Erlbaum.Child personality and temperament: Does the five-factor model embrace both domains?, 1994:323-338.

13. Markon KE, Krueger RF, Watson D, Delineating the structure of normal and abnormal personality: an integrative hierarchical approach, Journal of personality and social psychology, $2005 ; 88(1): 139-157$. https://doi.org/ 10.1037/0022-3514.88.1.139

14. Clark LA, Watson D, Temperament: a new paradigm for trait psychology. In: Pervin LA, John OP, editors. Handbook of Personality. 2nd ed. New York: Guilford ;1999: 399-423.

15. Markon KE, Krueger RF, \& Watson D, Delineating the structure of normal and abnormal personality:an integrative hierarchical approach, Journal of personality and social psychology, 2005;88(1):139157. https://doi.org/10.1037/0022-3514.88.1.139

16. Clark LA, Watson D, Temperament: a new paradigm for trait psychology. In: Pervin LA, John 
OP, editors. Handbook of Personality. 2nd ed. New York: Guilford; 1999: 399-423.

17. Kotov R, Gamez W, Schmidt F, et al, Linking "big" personality traits to anxiety, depressive, and substance use disorders: a meta-analysis, Psychological bulletin, 2010; 136(5):768-821. https://doi.org/10.1037/a0020327

18. Gramstad T.O., Gjestad R, Haver B, Personality traits predict job stress, depression and anxiety among junior physicians, BMC medical education, $2013 ; 13: 150$. https://doi.org/ 10.1186/1472-6920-13-150

19. Date C. van der Veen, Silvia DM, van Dijk,et al, The importance of personality and life-events in anxious depression: from trait to state anxiety, Aging \& Mental Health, 2017 ;21(11):1177-1183, DOI: 10.1080/13607863.2 016.1202894

20. Akiskal HS, Hirschfeld RM, Yerevanian BI, The relationship of personality to affective disorders, Archives of general psychiatry, 1983;40(7):801810 . https://doi.org/10.1001/ archpsyc.1983.01790060099013

21. Ormel J, Rosmalen J, Farmer A, Neuroticism: a non-informative marker of vulnerability to psychopathology, Social psychiatry and psychiatric epidemiology, 2004; 39(11): 906-912. https:// doi.org/10.1007/s00127-004-0873-y.

22. Klein DN, Kotov R, Bufferd SJ, Personality and depression: explanatory models and review of the evidence, Annual review of clinical psychology, 2011;7:269-295. https://doi.org/10.1146/annurevclinpsy-032210-104540

23. Ghorbani A, Hossein A, Teymor \& shayan, et al, Relationship between Type A Personality and Mental Health, Procedia - Social and Behavioral Sciences, 2011; 30:2010-2018.10.1016/ j.sbspro.2011.10.390.

24. Aftab A, Gehlot S, Joshi S, Evaluation of the relationship between Manas Prakriti, Deha Prakriti and Personality Traits".Thesis (MD) work in Dept. of Kriya Sharir, Faculty of Ayurveda, IMS, BHU, Varanasi; 2018.

25. Singh M, Stress scale, Institute of Research and Test Development, Andheri East, Mumbai; 2002.

26. Beck AT, Steer RA, Ball R, et al, Comparison of Beck Depression Inventories-IA and -II in psychiatric outpatients, Journal of personality assessment, 1996; 67(3):588-597.

27. Sinha WA, Sinha Anxiety Scale. 1996; University of Allahabad.

28. Ahmed Z, Julius S, Academic Performance, Resilience, Depression, Anxiety and Stress among Women College Students, Indian journal of positive psychology, 2015; 6:367-370

29. Srinivasan M, Reddy MM, Sarkar S, et al, Depression, Anxiety, and Stress among Rural South Indian Women-Prevalence and Correlates: A Community-Based Study, J Neurosci Rural Pract. 2020; 11(1):78-83.doi:10.1055/s-0039-1700595.

30. Sharma RK, \& Dash B. Charaka Samhita English translation. Reprint edition.Varanasi; Chowkhambha Sanskrit Series office, 2018: ISBN: 978-81-7080-012-9

31. Sharma R, Study of Manas Prakriti with Special reference to their vulnerability to Stress", World Journal of Pharmaceutical Research. 2018; 7(13): 619-626.

32. Das K, Li J, Fu G, et al,Genome-wide association studies for bivariate sparse longitudinal data. Human heredity.,2011;72(2):110-120. https:// doi.org/10.1159/000330781

33. Aktekin M, Karaman T, Senol YY, Anxiety, depression and stressful life events among medical students: a prospective study in Antalya,Turkey., Medical education, 2001; 35(1):12-17. https://doi.org/10.1046/ j.1365-2923.2001.00726.x

34. Waghachavare VB, Dhumale GB, Kadam YR, et al, A Study of Stress among Students of Professional Colleges from an Urban area in India, Sultan Qaboos University medical journal, 2001; 13(3):429-436

35. Kumari A, Jain J, Examination stress and anxiety: a study of college students, Global Journal of Multidisciplinary Studies, 2014; 04.

36. Inam B, Anxiety and Depression among Students of a Medical College in Saudi Arabia, International journal of health sciences, 2007;1:295-300.

37. Sahoo S, Khess CR, Prevalence of depression, anxiety, and stress among young male adults in India: a dimensional and categorical diagnosesbased study, The Journal of nervous and mental disease, 2010; 198(12):901-904 